\title{
Aproximación al aprendizaje desarrollador en la Educación Superior
}

\author{
Walfredo González Hernández* \\ Universidad de Matanzas
}

Resumo El aprendizaje desarrollador, como concepción de aprendizaje, es uno de los herederos más fieles de la concepción histórica y cultural de la psiquis humana. La propuesta más aceptada en este ámbito fue concebida para un contexto y un nivel educativo diferente al contexto universitario. En el artículo se revelan estas contradicciones y una propuesta conceptual de estas concepciones para el aprendizaje universitario.

PAlaVRaS-Chave: Aprendizaje desarrollador; Personalidad; Configuración. 


\title{
An approximation to the learning that's development a personality of student's on university
}

\begin{abstract}
The learning who's develop a personality of students, as learning conception, is one of the most faithful continuers of historical and cultural conceptions of human psyche. The most accepted proposal of this type of learning was conceived for a one context and one educational level different to the university context. In this paper is revealing these contradictions on the conceptions of the learning who's develop the personality of students and was proposed theorist conceptions of this type of learning to higher education.
\end{abstract}

KEYWORDS: Learn that's develop a personality; Personality;

Configuration.

\section{Introducción}

El proceso de aprender, que acompaña a los seres humanos desde el nacimiento, ha sido denominado aprendizaje. Desde la antigüedad hasta nuestros días se han producido las más variadas teorías para explicar cómo se produce este proceso, así como sus regularidades fundamentales. Si se centra en su origen entonces es posible analizar dos posiciones irreconciliables entre sí, los idealistas y los materialistas; por otro lado, si se asume una posición materialista es posible encontrar diferentes concepciones acerca del proceso. Para algunos autores este proceso era innato, o sea, sus regularidades, principios y formas de realizarlo estaban condicionados totalmente por la dotación genética del individuo. Posteriormente se fue variando esta posición y se analiza como un proceso de interacción con el medio en el cual el individuo actuaba en dependencia del sistema de influencias que se ejerciera sobre él. Esta cuestión planteaba al sujeto como un ente cuyas actuaciones no eran decididas y asumidas por él sino por el sistema de influencias que actuaran como desencadenantes de su conducta.

Posteriormente surgen otras concepciones que analizan al ser humano como procesador de información, el cual posee estructuras cognitivas que se ajustan y adaptan al medio ambiente. De esta manera el individuo establece relaciones de adaptación y sensibilización con el medio ambiente en el cual debe existir una relación de equilibrio. Otra concepción aborda al ser humano como una singularidad única e irrepetible, en relación consigo mismo y sus tendencias de autorrealización. Estas visiones sobre el ser humano han impactado de manera importante en las explicaciones sobre el aprendizaje.

Otra de las concepciones acerca del ser humano enfatiza que

... para explicar las formas más complejas de la vida consciente del hombre es imprescindible salir de los límites del organismo, buscar los orígenes de esta vida consciente y del comportamiento "categorial" no en las profundidades del cerebro ni en las profundidades del alma, sino en las condiciones externas de la vida y, en primer lugar, 
de la vida social, en las formas histórico sociales de la existencia del hombre. (Corrales et al., 2016, p. 22).

Para esta concepción el hombre es un ser biológico en la medida que tiene una dotación genética, psicológico en tanto es portador de una psiquis y social en la medida que se desarrolla en una determinada sociedad. El ser humano, tal y como es conocido hoy, ha devenido en un largo proceso de transformaciones fisiológicas y psíquicas. Sin embargo, uno de los aspectos que lo ha caracterizado desde los primeros homínidos ha sido el aprendizaje con un marcado carácter social. El hombre desde su nacimiento está condicionado socialmente dado por su relativa poca cantidad de reflejos incondicionados que posee y lo hace dependiente de quien lo crie, de ahí la plasticidad del órgano principal de la actividad psíquica: el cerebro. De esta manera, la cría humana nace condicionada para adquirir nuevas experiencias y estructurar nuevas necesidades si se satisfacen las orgánicas. Quiere decir que la cría humana nace para aprender.

En esta concepción materialista del aprendizaje fundada por el trío Vygotsky - Luria - Leontiev, pero continuada por múltiples autores (González Rey, 2012), se reconoce el carácter materialista y social del psiquismo humano. Una de las expresiones de esta concepción del desarrollo humano que ha marcado pautas hoy en Cuba en el plano didáctico es el aprendizaje desarrollador. Sin embargo, esta conceptualización del aprendizaje basado en los pilares de los supuestos teóricos de la personalidad como motivación, regulación y significatividad no puede explicar estos procesos de la realidad teniendo en cuenta la categoría situación social de desarrollo en la cual se encuentran los estudiantes universitarios. Los elementos estructurales y dinámicos que componen la personalidad en las explicaciones del aprendizaje desarrollador, como está reflejada en la literatura actual (Zilberstein e Olmedo, 2014; Núñez, 2015; Sospedra e Rosa, 2015; Cañarte et al., 2016) aun cuando investigan el desarrollo en jóvenes, no se corresponden con la juventud, situación social de desarrollo por la cual transitan los estudiantes universitarios. Es válido resaltar la misión formativa de los estudios universitarios de integrar a la sociedad un profesional competente con un alto compromiso social que le permita enfrentar los desafíos de su profesión.

Es por ello que este artículo pretende de una manera suscita develar las regularidades, que deben caracterizar un aprendizaje desarrollador en los jóvenes que asisten a la universidad como centro formativo de futuros profesionales. En un primer momento se caracterizará cómo se analiza el desarrollo humano desde la concepción histórica cultural actual. Posteriormente se analizarán los postulados esenciales del llamado aprendizaje desarrollador y, por último, aquellas categorías esenciales de la psicología de orientación dialéctico materialista que se asumen como importantes para conceptualizar el aprendizaje desarrollador en la formación de profesionales desde las universidades.

\section{Desarrollo}

Para (Vygotsky, 1995) no es cualquier enseñanza la que produce el desarrollo, es la que toma en cuenta las potencialidades del alumno en cada momento y se instrumenta sobre lo que ha adquirido, pero esencialmente sobre lo que debe adquirir, 
por eso se considera una enseñanza hacia el futuro no sólo tomando en cuenta el presente del desarrollo. En su obra señalaba:

La mayoría de las investigaciones que tienen que ver con el aprendizaje escolar miden el nivel de desarrollo mental del niño y le hacen solucionar determinados problemas estandarizados. Se supone que el problema que puede resolver por sí solo indica el nivel de su desarrollo mental en ese momento. (Vygotsky, 1995, p. 78).

Pero de este modo queda así clara la posición de Vigotsky con respecto a la relación enseñanza aprendizaje, en la que continúa profundizando, cuando señala: "En el desarrollo del niño... la imitación y la instrucción desempeñan un papel fundamental, descubren las cualidades específicamente humanas de la mente y conducen al alumno a nuevos niveles de desarrollo" "Cita” (Vygotsky, 1995, p. 79) reafirmando su postura desarrolladora.

Diversos autores cubanos buscan dar respuesta a los procesos de aprendizaje y desarrollo a partir de la elaboración de estrategias, procedimientos, exigencias, tareas de aprendizaje, que en el orden didáctico, enriquezcan la enseñanza que se instrumente para el alcance de este objetivo, en las que el alumno participe en un proceso donde puedan ser desarrolladas al máximo sus potencialidades (Coon e Mitterer, 2012; Suárez et al., 2013; López et al., 2016; Sarría e Fernández, 2017)

dizaje humano es:

En consonancia con lo anterior se asume por diversos autores que el apren-

El proceso dialéctico de apropiación de los contenidos y las formas de conocer, hacer, convivir y ser construidos en la experiencia socio histórica, en el cual se producen, como resultado de la actividad del individuo y de la interacción con otras personas, cambios relativamente duraderos y generalizables, que le permiten adaptarse a la realidad, transformarla y crecer como personalidad. (Castellanos, 2005, p. 7).

El profesor tiene la responsabilidad de enseñar a aprender, fundamental en la formación de profesores. Debe propiciar la participación del estudiante y proporcionarle la oportunidad de aplicar, en condiciones apropiadas, lo que aprende en la escuela. Debe esforzarse para que el estudiante tenga éxito desde el principio, llevarlo a amar y apreciar lo que aprende, estimular el éxito del estudiante.

De tal manera se considera por los autores consultados (Díaz Lara e Suárez Suárez, 2014; De La Cruz Rodríguez, 2016; Kanhime e González, 2017) que es importante que el profesor conozca las motivaciones e intereses de los estudiantes, que sea capaz de percibir el momento acertado para enseñar, cuando el estudiante está en disposición de aprender y procurar que el aprendizaje sea significativo para él, cuestión importante en criterio del autor, de tal manera que el aprendizaje conduzca al desarrollo (Castellanos, 2001; Castellanos Simons, 2003).

Para varios autores (Suárez et al., 2013; Trimiño Quiala, 2013) aprendizaje desarrollador es el proceso de apropiación por el alumno de la cultura bajo condiciones de orientación e interacción social. Hacer suya esa cultura, requiere de un proceso 
activo, reflexivo, regulado, mediante el cual aprende, de forma gradual, acerca de los objetos, procedimientos, las formas de actuar, las formas de interacción social, de pensar, del contexto histórico social en el que se desarrolla y de cuyo proceso dependerá su propio desarrollo; con un objetivo fundamental de manera explícita: el desarrollo de la personalidad del estudiante.

Para (Ginoris, 2009; Núñez, 2015; Sospedra e Rosa, 2015) lograr en los estudiantes un aprendizaje desarrollador es necesario trabajar por cualidades como las siguientes:

El aprendizaje debe distinguirse por ser activo y regulado, para lo cual el estudiante tiene que ser constructor de su propio conocimiento y protagonista en el PEA, para lo cual se requiere lograr en los estudiantes la aplicación creadora y la transferencia de conocimientos y habilidades a situaciones docentes nuevas, lo que se traduce en aprendizaje como producción de sus propios y nuevos saberes, incluyendo en los mismos la actividad metacognitiva del estudiante en su aprendizaje desarrollador.

Esta cualidad metacognitiva de un aprendizaje desarrollador se expresa en dos funciones: el aprendizaje es, además de activo, una reflexión metacognitiva que deviene aprendizaje autorregulado y conduce a la comprensión por el estudiante de las cualidades que distinguen el aprendizaje propio, las alternativas para producir sus conocimientos. Es decir, conocimientos sobre su propio proceso cognoscitivo: metaconocimientos. De ahí la necesidad de aprender a aprender.

Para (Ginoris, 2009; Zilberstein e Olmedo, 2014)se refieren también a los cambios de roles de los protagonistas en el proceso de enseñanza aprendizaje desarrollador, donde la actividad de enseñar que lleva a cabo el maestro debe estar caracterizada por:

- La creación de un ambiente afectivo en la clase donde se estimule y refuerce la participación permanente de los estudiantes en su aprendizaje.

- La estimulación del comportamiento grupal de los estudiantes.

- La orientación y vinculación de los objetivos formativos e instructivos.

- La atención a las diferencias individuales sin dejar de considerar las cualidades grupales.

- Las relaciones e integración de las diferentes asignaturas y entre estas y la realidad, entre otras.

Otros autores (Cruz et al., 2010; Zilberstein e Olmedo, 2014) coinciden en afirmar que le corresponde al estudiante asumir el lugar de protagonista y sujeto en el proceso, tornándose constructor y reconstructor de sus saberes, desarrollar un pensamiento analítico, reflexivo, crítico y alternativo que se materialice en un nuevo estilo de aprendizaje, de manera que el proceso cognitivo se transforme de reproductivo, concreto y situacional en uno productivo, generalizador y conceptual, construir para sí, además de conocimientos sobre el mundo externo y objetivo, conocimientos sobre su aprendizaje y su propia personalidad, necesidades, vías y formas de actuar (metaconocimientos), entre otras cuestiones. 
Se asume que el proceso de aprendizaje desarrollador constituye la vía esencial para la apropiación de conocimientos, habilidades, normas de relación emocional, de comportamientos y valores legados por la humanidad, que se expresan en el contenido de enseñanza, en estrecho vínculo con el resto de las actividades docentes y extra docentes que realizan los estudiantes. En la literatura al respecto se asumen el conjunto de dimensiones y subdimensiones tratados que se expresan en la siguiente figura:

Figura 1 - Dimensiones y subdimensiones del aprendizaje desarrollador (Castellanos Simons, 2003, p. 10).

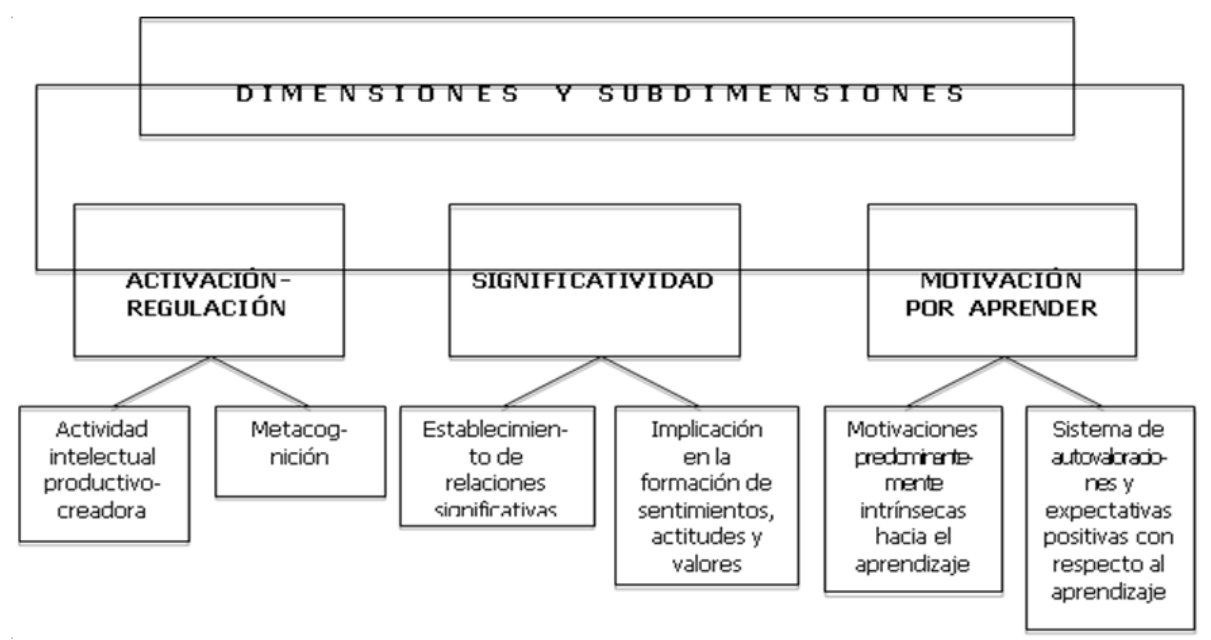

Sin embargo, es necesario apuntar algunos elementos que no quedan suficientemente claros en las investigaciones anteriores ni en aquellas que se refieren a este aprendizaje en las universidades (Coon e Mitterer, 2012; Castellanos e Caballero, 2016) por lo que se aprecia que no se considera en la concepción del aprendizaje desarrollador la categoría situación social de desarrollo en su explicación del aprendizaje.

La categoría situación social de desarrollo (SSD) es una de aquellas que deben ser tenidas en cuenta al explicar las regularidades del aprendizaje. En (Fariñas, 2005, p. 57) se resume

...al principio de cada período etario se establece una relación peculiar, única e irrepetible, específica para esa etapa de la vida, entre el niño y su medio, ante todo social. A esta relación la denominamos situación social del desarrollo...esta relación significa... un nuevo carácter de la percepción de la realidad externa y de la actividad en ésta, un nuevo carácter de la percepción de la vida interna del propio niño y de la activación interna de su vida psíquica... La situación social del desarrollo es el momento de partida para todos los cambios dinámicos, que ocurren en el desarrollo durante un período dado. Ella determina de manera completa y global aquellas formas y aquel camino, a través de los cuales el niño adquiere nuevas propiedades de la personalidad. 
Siguiendo esta idea otra autora (Fariñas, 2005, p. 57)

... el desarrollo consiste entonces en la llegada a una nueva situación
social del desarrollo, en la que se genera y sostiene el desenvolvi-
miento humano ulterior... La situación social del desarrollo expresa
la conjunción dinámica de las condiciones interpersonales o exter-
nas y las intrapersonales o internas, que producen el desarrollo de la
persona... que también forma parte de dichas vivencias personales.

Se infiere entonces que si el aprendizaje debe conducir al desarrollo debe tener en cuenta las características esenciales de cada nueva periodización, en este caso la juventud como edad psicológica. En esta edad comienza a delinear su sentido de la vida, como conjunto de objetivos mediatos que se traza en las cuales se van entrelazando las diferentes esferas de significación para la personalidad y requieren de la elaboración de una estrategia encaminada, a emprender acciones en el presente, que contribuyan al logro de metas futuras. Es en esta edad cuando se comienza a elaborar el proyecto de vida y se estructuran las representaciones sobre cuál tipo de actividad científico-profesional o laboral va a dedicarse, y en consonancia con esta decisión, organizar su comportamiento, esta decisión deviene en la actividad rectora que va a desarrollar en esta etapa. En este artículo se referirá a la actividad profesional para lo cual el ente formativo por excelencia es la universidad. Es por ello que asumir solamente la motivación como eje estructural de lo afectivo para esta etapa restringe las formaciones que conducen al desarrollo de la personalidad.

Con la aportación de las nociones de ley del desarrollo psíquico superior desarrollo próximo, el enfoque histórico-cultural aborda el desarrollo humano desde sus condiciones concretas de existencia materiales-espirituales- psicológicas (SSD) y el papel de los mediadores -otros significativos y productos culturales- en la construcción dinámica de sentido y proyectos de vida de la persona.

Por lo tanto, se asume que uno de los ejes articuladores en la dinámica de desarrollo para la juventud es el proyecto de vida “... una estructura psicológica que expresa las direcciones esenciales de la persona, en el contexto social de relaciones materiales y espirituales de existencia que determinan una sociedad concreta" "Cita" (D’Angelo, 2004, p. 6) y cuya formación es “... a partir de la "posición externa” del individuo y la configuración de su experiencia personal, las posibilidades o recursos disponibles, el sistema de necesidades, objetivos y aspiraciones y las orientaciones (o actitudes) y valores vitales de la persona" "Cita" (D’Angelo, 2004, p. 8). Esta categoría estructurada por D’Angelo (2004) permite comprender mejor la estructuración afectiva, cognitiva y reguladora de la personalidad en la juventud y que permite estructurar acciones educativas para el tránsito hacia la adultez además de incluir la motivación como elemento constituyente (Pachón et al., 2016).

Al hablar de la motivación como parte del proyecto de vida no se refiere a cualquier motivación. En este sentido, es importante destacar como parte del proyecto de vida las denominadas tendencias orientadoras de la personalidad (González, 2000; González Rey, 2015), expresando aquellas motivaciones que orientan la actividad de la personalidad, a las cuales se les debe prestar especial atención desde su aparición y sobre todo en la formación de profesionales. Es por ello que el proceso de enseñanza 
aprendizaje de profesionales será verdaderamente desarrollador en cuanto satisfaga las contradicciones fundamentales contenidas en las tendencias orientadoras de las personalidades relacionadas con el ejercicio de su profesión cuando esta forma parte de su proyecto de vida.

Al asumir esta concepción de aprendizaje en el cual el proyecto de vida se torna una de sus dimensiones es importante destacar sus elementos constitutivos (D’Angelo Hernández, 2002, p. 8):

- Orientaciones de la personalidad (Valores morales, estéticos, sociales, etc. $\mathrm{y}$ fines vitales)

- Programación de tareas-metas vitales-planes-acción social.

- Autodirección personal: Estilos y mecanismos psicológicos de regulación y acción que implican estrategias y formas de autoexpresión e integración personal y autodesarrollo.

El interés pedagógico de semejante perspectiva puede ser importante, en tanto provee de una comprensión holística, dinámica y contradictoria de las articulaciones complejas del individuo y su contexto social mediato e inmediato. En esta perspectiva en que las acciones educativas tienen que tomar el referente de los procesos psicológicos que se recortan en la dinámica mayor de las situaciones sociales y de las condiciones de la praxis individual-social (D’Angelo Hernández, 2002).

Otro de los elementos esenciales en el proceso de aprendizaje es la unidad de lo cognitivo y lo afectivo que se expresa en el sentido. En Pensamiento y Lenguaje, se define sentido

... el agregado de todos los elementos psi`cológicos que emergen en nuestra conciencia como resultado de la palabra. El sentido es una formación dinámica fluida y compleja que tiene varias zonas que varían en su estabiliาdad. El significado es apenas una de esas zo $\neg$ nas de sentido que la palabra adquiere en el contexto del habla (Vygotsky, 1995, p. 275 y 276).

Esta definición explicita la importancia de una unidad de análisis de la vida psíquica en la cual se integra todos los elementos psicológicos dentro de los cuales se encuentran aquellos que intervienen en un proceso de aprendizaje. Sin embargo, esta categoría no aparece en la literatura consultada sobre aprendizaje desarrollador y en la obra de la autora principal sobre el tema aparece solamente para explicar la significatividad del aprendizaje. Se considera que esta es una limitación esencial en la concepción actual cuando se plantea

$$
\begin{aligned}
& \text {... un aprendizaje significativo es aquel que, partiendo de los conoci- } \\
& \text { mientos, actitudes, motivaciones, intereses, y experiencia previa del } \\
& \text { estudiante hace que el nuevo contenido cobre para él un determina- } \\
& \text { do sentido (Castellanos e Caballero, 2016, p. 23). }
\end{aligned}
$$

En esta concepción del aprendizaje es el objetivo y debe ser el punto de partida del aprendizaje para entrelazar toda la dinámica de los elementos psicológicos que deben intervenir en el aprendizaje con el nuevo contenido. Siguiendo con esta idea, la consideración de la categoría sentido subjetivo 


\begin{abstract}
...una unidad simbólico-emocional que se organiza en la experiencia social de la persona, en la cual la emergencia de una emoción estimula una expresión simbólica y viceversa, en un proceso en que se definen complejas configuraciones subjetivas sobre lo vivido, que representan verdaderas producciones subjetivas, en las cuales la experiencia vivida es inseparable de la configuración subjetiva de quien las vive (González-Rey, 2010, p. 233).
\end{abstract}

Esta concepción amplía el sentido del aprendizaje desde lo individual hasta lo social en un sentido cultural e histórico que debería ser tomado en cuenta en toda su amplitud para definir el aprendizaje. Por tanto, es importante destacar que el aprendizaje no debe ser caracterizado como significativo sino subjetivado, en función de la integración su subjetividad con los objetivos sociales para los cuales se produce el aprendizaje. Esta concepción del aprendizaje se aprecia más dinámica e integradora que la asumida en el aprendizaje desarrollador abordada hasta el momento en la literatura al respecto. Quiere ello decir, que un aprendizaje que oriente y cree sentidos subjetivos en los estudiantes es un aprendizaje que logra el desarrollo de la subjetividad humana entendida como "...como la producción simbólico-emocional que emerge ante una experiencia vivida, la cual integra lo histórico y lo contextual en el proceso de su configuración. La unidad básica de la subjetividad son los sentidos subjetivos" "Cita” (González Rey, 2015, p. 15). Este tratamiento de la subjetividad asume las configuraciones, formaciones y unidades psíquicas en un sentido integrador, holístico y complejo en el cual se reconocen la historicidad del aprendizaje y su proyección futura, además del momento presente, cuestiones estas esenciales para una aplicación consecuente de la zona de desarrollo próximo en la formación de profesionales. También abarca aspectos esenciales como la búsqueda de estrategias y métodos para la apropiación y búsqueda de los contenidos propios de la educación superior con un alto nivel de generalización y abstracción que les servirán de base para la actualización propia de la etapa adulta que son típicas de la juventud.

En búsqueda de una explicación sobre las configuraciones en la subjetividad se han encontrado diversas aproximaciones sobre este término. Para González Rey (2010) en el orden psicológico define configuración “... como sistema de formaciones diferentes, donde un mismo elemento psicológico parcial puede aparecer de forma simultánea en unas u otras de dichas formaciones, incluso con un sentido psicológico diferente." “Cita” (González-Rey, 2010, p. 245). De esta definición se infieren elementos importantes de la organización de la configuración. Uno de ellos es el sistema de formaciones y se infiere que depende de determinadas situaciones cuando continúa expresando "...la naturaleza configuracional de la personalidad radica en la integración de contenidos psicológicos cognitivos y afectivos en una nueva realidad" "Cita" (González-Rey, 2010, p. 246). Esta última afirmación vincula la configuración a la realidad sin especificar cuáles son los aspectos de la realidad que originan su organización.

Sin embargo, cuando se refiere a la personalidad como “... una configuración de contenidos subjetivos que tienen una real significación personal” "Cita” (González_Rey, 2016, p. 4), aportando el por qué la configuración adquiere estas características, como en este caso la personalidad, en dependencia de la significación que la realidad y que en su interacción con ella adquiere. En los autores analizados anteriormente no explican cómo se estructura la relación con la realidad ni cuáles son los procesos que 
intervienen en la determinación de las configuraciones. En este artículo, considerando los aspectos ya planteados, se define la configuración como la estructura en que un sistema complejo organiza o reorganiza a sus componentes (estructurales y/o funcionales) para interactuar con otros sistemas, en dependencia de sus características propias y del otro sistema, así como de la naturaleza de la interacción entre ellos. Se puede aseverar entonces, que un sistema complejo puede adquirir varias configuraciones en su interacción con otros sistemas, permitiéndole interactuar en dependencia de sus condiciones internas y las externas con un máximo de adaptabilidad y flexibilidad. Por ende, todos los sistemas complejos son sistemas configuracionales.

De la definición anterior se desprende que el aprendizaje es una configuración de la subjetividad como sistema complejo que interactúa con un conjunto de sistemas externos entre los cuales se pueden señalar el sistema escolar y el saber. E1 aprendizaje, concebido de esta manera, se configura en el acto de aprender; en el cual se entrelazan sus sentidos subjetivos ya que debe tomar decisiones sobre este proceso, confrontarse con el otro, revivir situaciones vividas con cargas positivas, negativas y/o ambivalentes que toman nuevas formas en sus expresiones al mismo tiempo que se cumple con el objetivo social de la enseñanza. En este proceso de aprendizaje la imaginación, como producción subjetiva (González Rey e Patiño Torres, 2017), juega un papel esencial como fuente de ideas en las cuales se expresan sus producciones subjetivas integradoras y abarcadoras. Por ende, entonces el aprendizaje es también un proceso productor de ideas en el cual el sujeto reelabora los contenidos de su personalidad si en este proceso se alimentan sus tendencias orientadoras. Entonces no es el aprendizaje solamente autorregulado - activado, sino que es un proceso configuracional de la subjetividad humana. Esta concepción del aprendizaje integra no sólo la actividad, la regulación y la autorregulación sino otros procesos productivos de la subjetividad humana como la imaginación y los procesos intuitivos que intervienen en esta (Absatova et al., 2014; Antonio et al., 2014; Aras, 2015; Berdibayeva et al., 2015). La tendencia a la búsqueda de problemas y su solución es una de las características distintivas de la juventud como edad psicológica que aparece en la concepción de aprendizaje desarrollador que se propone en este artículo.

En esta edad las actividades seleccionadas tienen una mayor intencionalidad sobre la base de su proyecto de vida, por lo cual el sistema de interacciones a las cuales se entrelazan tienen mayor apertura y diversidad que en edades anteriores; y estas peculiaridades deben ser reflejadas en la teoría didáctica. Siguiendo este orden de ideas, entonces se reconoce que el aprendizaje es un proceso que se basa en la no linealidad de las interacciones entre los componentes del proceso de enseñanza - aprendizaje y de éstos con la comunidad en el sentido más amplio posible. Entre ellos se entretejen relaciones nada simples, que pueden constituir una red de relaciones abiertas con otros subsistemas a los cuales se integran configurando sistemas cada vez más complejos.

En la teoría didáctica derivada de una concepción histórico - cultural no se es lo suficientemente claro al abordar la relación entre la complejidad del contexto socio - histórico y la propia de la psiquis del estudiante que se expresa en la subjetividad (Rey, 2014; 2015; 2016; González Rey e Patiño Torres, 2017). Esta relación expresada anteriormente en la Didáctica no es un problema suficientemente resuelto. Se coinci- 
de con este autor que es un proceso totalmente alejado del equilibrio al constituir un reflejo subjetivo del contexto socio histórico totalmente diferente para cada estudiante. En dinámica de un proceso general en el cual confluyen un conjunto de estudiantes, las estructuras grupales en las cuales se organiza el aprendizaje y los profesores por un lado para el logro de la formación profesional en el contexto universitario y, por otro, las subjetividades de los estudiantes desarrolladas en su devenir histórico se expresa una de las contradicciones fundamentales de la didáctica. Esta contradicción se enmarca en el contexto escolar; sin embargo, otra contradicción se aprecia entre las necesidades sociales de formación del profesional y la obsolescencia de la formación universitaria cuando egresan y se insertan en los procesos organizacionales. Existen acercamientos teóricos para la solución a las dos contradicciones planteadas anteriormente, sin embargo, no se observan con amplitud las categorías psicológicas tratadas anteriormente como parte del enfoque histórico - cultural.

No se asume en la teoría didáctica actual que en el proceso de aprendizaje el estudiante interactúa con otros actores ajenos al proceso y que intervienen en el mismo de manera diversa y, en ocasiones contradictorias, con el proceso formativo. Ello lleva a pensar que en vez de una interacción entre la estructura formativa y estos actores ajenos debe analizarse como un equilibrio entre estos sistemas dinámicos: la sociedad y la universidad como parte de ella, con características de no linealidad, dinamismo y abertura a otros sistemas para lograr el desarrollo de la personalidad del individuo.

Una idea no presente en el contexto educativo sobre aprendizaje se encuentra en la multiculturalidad presente en la sociedad actual, con gran influencia de las redes informáticas que permiten la interacción social en modelos de comunicación y desarrollo más holísticos e integradores como el modelo de (Zeidmane e Duka, 2014) basado en el enfoque histórico cultural, que amplía y enriquece. Desde esta perspectiva, la relación universidad - sociedad ya no es tan lineal, coherente y exenta de contradicciones como se expresan en los libros y artículos de Didáctica consultados, ya que la interacción de los participantes en el proceso con la sociedad puede darse en dos sociedades contradictorias entre sí. De esta manera, en un mundo cada vez más interconectado las interacciones entre la escuela - sociedad deben ser flexibles, tolerantes a las emergencias y, sobre todo abiertas a la interacción con otros sistemas tan dinámicos y no lineales como ellos, como puede ser el contexto internacional. Por tanto, el contexto en el cual transcurre el aprendizaje cumple con las características esenciales de los sistemas complejos:

- Las funciones de los elementos (subsistemas) del sistema no son independientes; esto determina la interdefinibilidad de los componentes;

- El sistema como totalidad es abierto, es decir, carece de fronteras rígidas; está inmerso en una realidad más amplia con la cual interactúa por medio de flujos de materia, energía, recursos económicos, políticas regionales, nacionales, entre otras.

- Alto grado de indeterminación y de improbabilidad (Eduardo Maldonado, 2014, p. 12).

Llevando a afirmar que, desde la visión de la relación universidad y sociedad, el aprendizaje es un proceso de interacciones y contexto de orden complejo. 
Siguiendo este análisis, ahora en el orden psíquico, también es el aprendizaje un proceso complejo. Para este análisis se considera indispensable partir de uno de los procesos que intervienen en el aprendizaje y ha sido poco estudiado desde la concepción histórica y cultural: la intuición.

Desde una perspectiva histórica cultural se destaca la intuición como parte de los procesos del pensamiento íntimamente relacionados los procesos afectivos. Se destaca como parte de la relación dialéctica que se establece con el pensamiento lógico, vital para el desarrollo de la creatividad (Rodrigues, 2011; Hernández, 2013). Aunque no se constata que (3) se haya ocupado de la intuición se pueden atisbar en su obra elementos que pudieran conducir a explicar el origen de las asociaciones libres expresadas anteriormente cuando expresa el concepto de pensamiento por complejos diferente al pensamiento conceptual. De las concepciones expresadas se puede inferir que existe gran similitud entre las asociaciones divergentes (Sinclair, 2011) y el pensamiento por complejos (Vygotsky, 1995).

Continuando la idea anterior en la obra de Vygotsky, se va transitando hacia un pensamiento conceptual que, sin embargo, en este artículo se asume que no elimina el pensamiento por complejos, sino que este subyace conformando el lenguaje interiorizado. Gran parte de los autores dedicados a la intuición (Pretz, 2011) lo explican de manera similar, como un proceso del cual se debe tomar nota inmediata para no perder las ideas que se expresan. La principal limitante de Vygostky está en cómo desarrollar la intuición tomando como base sus ideas. Como se puede apreciar en este bosquejo sobre las concepciones fundamentales acerca de la intuición se trata de un proceso multifactorial con causas que aún no han sido totalmente esclarecidas sobre bases científicas. Se infiere además del bosquejo anterior que es un proceso con un alto grado de incertidumbre que involucra los procesos no conscientes. Es por ello que este autor considera que es un proceso caracterizado por la complejidad. Otro elemento a tener en cuenta es la relación intuición y los procesos subjetivos que tiene lugar en la persona. Es por ello que se considera la intuición como un proceso que forma parte del sentido subjetivo al desarrollarse sobre la base de la experiencia vivida y adoptando formas dinámicas. Se infiere entonces que la intuición se manifiesta en dependencia de los sentidos subjetivos del individuo constituyendo una expresión de la subjetividad de la persona (González-Rey, 2010; Martínez, 2010) en una actividad determinada, por lo cual se manifiesta de manera configuracional. De ahí que la intuición se regula por la personalidad, aunque se realiza a nivel no consciente. Conjuntamente con la imaginación ya tratada anteriormente, la intuición es el otro pilar para la producción de ideas.

Por ende, después del análisis realizado, se entiende que el aprendizaje en la educación superior debe ser subjetivo y complejo por las formas de apropiación del conocimiento y los objetivos del mismo. Al mismo tiempo, es configuracional por la manera en que se estructura en su dinámica. Es entonces que se asume el aprendizaje desarrollador como un proceso complejo que implica al sistema de sentidos subjetivos asociados con la apropiación del contenido de enseñanza, sobre cómo se expresan sus configuraciones subjetivas en la rama del saber humano con sus formas de actividad fundamentales, en interacción no lineal con el resto de los sistemas que intervienen. 
Esta definición incluye la posibilidad de integrarse a las redes sociales que se entretejen actuando en consonancia con sus tendencias orientadoras y proyecto de vida relacionados con la profesión. Debe, además, socializar los resultados de este proceso dentro de un contexto social en el que descubre relaciones complejas en el proceso de apropiación de los contenidos con su introducción en diversas áreas del conocimiento humano.

Esta definición de aprendizaje desarrollador se considera elementos más integradores y holísticos e involucra todos los elementos estructurales y dinámicos de la personalidad del sujeto que se forma para el ejercicio de una profesión. Constituye una concepción optimista del aprendizaje en cuanto expresa los elementos pasados, presentes y, lo que la diferencia cualitativamente de la anterior, se proyecta hacia el futuro teniendo en cuenta las diferentes SSD. Esta definición trasciende el marco de la enseñanza universitaria y es posible aplicarla a los procesos formativos que se dan en el orden de la formación continua.

\section{Conclusiones}

Las concepciones vygotskianas sobre aprendizaje han revolucionado las posiciones actuales sobre el aprendizaje desde procesos objetivos y subjetivos que les permite una visión histórica y cultural sobre este proceso. El aprendizaje desarrollador adolece de insuficiencias en su concepción teórica para explicar cómo transcurre el aprendizaje cuando se expresan en su dinámica y estructura formaciones más complejas e integradoras que la motivación, la significatividad y la regulación. Considerar al aprendizaje desarrollador desde configuraciones subjetivas permite una visión más actual, holística y real del proceso de apropiación de contenidos desde la situación social de desarrollo, integrando las diversas categorías del enfoque dialéctico materialista del desarrollo de la psiquis.

\section{Referências}

ABSATOVA, M. A. et al. Personal Peculiarities of Gifted Children Development. Procedia Social and Behavioral Sciences, v. 136, p. 400-404, 2014. ISSN 18770428.

ANTONIO, T. et al. Correlations Creativity, Intelligence, Personality, and Entrepreneurship Achievement. Procedia - Social and Behavioral Sciences, v. 115, p. 251-257, 2014. ISSN 18770428.

ARAS, G. Personality and Individual Differences: Literature in Psychology- Psychology in Literature. Procedia - Social and Behavioral Sciences, v. 185, p. 250-257, 2015. ISSN 18770428.

BERDIBAYEVA, S. et al. Psychological Features of Gender Relations in Self-regulation of Personality. Procedia - Social and Behavioral Sciences, v. 171, p. 203-208, 2015. ISSN 18770428.

CAÑARTE, J.; QUEVEDO, N.; GARCÍA, N. Una estrategia para el aprendizaje desarrollador del inglés en el estudiante de carreras técnicas con la modalidad combinada. Revista Magazine de las Ciencias. ISSN 2528-8091, v. 1, n. 1, p. 51-60, 2016. ISSN 2528-8091.

CASTELLANOS, D. Aprender y enseñar en la escuela. Ciudad de la Habana: Editorial Pueblo y Educación, 2001.

CASTELLANOS, D. Estrategias para promover el aprendizaje desarrollador en el contexto escolar. Pedagogía 2005. EDUCACIÓN, E. P. Y. Ciudad de la Habana. 22005. 
CASTELLANOS, R.; CABALLERO, B. Acceso y calidad. Educación media y educación superior una articulación necesaria. Cambios y Permanencias, n. 5, p. 468-485, 2016. ISSN 2027-5528.

CASTELLANOS SIMONS, D. Estrategias para promover el aprendizaje desarrollador en el contexto escolar. La Habana. : Editorial Félix Varela, 2003.

COON, D.; MITTERER, J. Psychology Modules for Active Learning. Brock, England: Cengage Learning Customer \& Sales Support, 2012.

CORRALES, L.; HERRERA, J.; MARTÍN, D. La superación profesional de los docentes universitarios para el perfeccionamiento de la dirección del aprendizaje. Márgenes, v. 3, n. 3, p. 327-346, 2016.

CRUZ, A. V.; DIÉGUEZ, O.; CARBONELL, E.. La tarea docente. Una alternativa desarrolladora para estimular el aprendizaje del idioma inglés. Cuadernos de Educación y Desarrollo, n. 22, 2010.

D’ANGELO HERNÁNDEZ, O. Proyecto de vida y desarrollo integral humano. Revista Internacional Crecemos, v. 6, n. 2, 2002.

D’ANGELO, O. Proyecto de vida como categoria básica de interpretación de la identidad individual y social. Centro de Investigaciones Psicológicas y Sociológicas. La Habana, p.23. 2004

DE LA CRUZ RODRÍGUEZ, R. D. Teorías implícitas sobre evaluación en matemáticas que poseen los docentes en formación inicial de las universidades de extremadura España y trujillo Perú. 2016.1 - 138 (Máster Oficial Interuniversitario de Investigación en la Enseñanza y el Aprendizaje de las Ciencias Experimentales, Sociales y Matemáticas Especialidad: DIDÁCTICA DE LAS MATEMÁTICAS). Facultad de Educación, Universidad de Extremadura, Badajoz, España.

DÍAZ LARA, L.; SUÁREZ SUÁREZ, G. Necesidad de promover el aprendizaje desarrollador en estudiantes universitarios. Universidad y Sociedad, v. 5, n. 3, 2014. ISSN 2218-3620.

EDUARDO MALDONADO, C. ¿Qué es eso de Pedagogía y Educación en la complejidad? Intersticios Sociales, n. 7, 2014.

FARIÑAS, G. Acerca del modelo interdisciplinario sobre el desarrollo humano. Psicología, Educación y Sociedad. Un estudio sobre el desarrollo humano. La Habana: Félix Varela, 2005.

GINORIS, O. Fundamentos didácticos de la educación superior cubana. La Habana: Editorial Félix Varela, 2009. 251.

GONZÁLEZ-REY, F. Las categorías de sentido, sentido personal y sentido subjetivo en una perspectiva histórico-cultural: un camino hacia una nueva definición de subjetividad. Universitas Psychologica, v. 9, n. 1, p. 241 - 253, 2010.

GONZÁLEZ, F. L. S. Vygotski y el problema de la personalidad en el enfoque histórico-cultural. III Conferência de Pesquisa Sócio-cultural. Campinas, Sâo Paulo. Cultura - A dimensão psicológica e a mudança histórica e cultural 2000.

GONZÁLEZ REY, F. Reflexões sobre o desenvolvimento da psicologia soviética: Focando algumas omissões da interpretação ocidental. Psicologia \& Sociedade, v. 24, n. 2, p. 263-271, 2012.

GONZÁLEZ REY, F. Human Motivation in Question: Discussing Emotions, Motives, and Subjectivity from a Cultural-Historical Standpoint. Journal for the Theory of Social Behaviour, v. 45, n. 4, p. 4, 9-439, 2015.

GONZÁLEZ REY, F.; PATIÑO TORRES, J. F. La Epistemología Cualitativa y el estudio de la subjetividad en una perspectiva cultural-histórica. Conversación con Fernando González Rey. Revista de Estudios Sociales No. 35, v. 60, p. 120-127, 2017. ISSN 0123885X-19005180. 
GONZÁLEZ_REY, F. Vygotsky's Concept of Perezhivanie in The Psychology of Art and at the Final Moment of His Work: Advancing His Legacy. Mind, Culture, and Activity, v. 23, n. 4, p. 305-314, 2016. ISSN 1074-9039.

HERNÁNDEZ, W. G. Creativity Development in Informatics Teaching Using the Project Focus. iJEP, v. 3, n. 1, p. 22 - 30, 2013

KANHIME, M.; GONZÁLEZ, W. Estrategia metodológica para lograr la evaluación desarrolladora de la matemática en la escuela de formación de profesores de Kuando Kubango, Angola. Góndola, Enseñanza y Aprendizaje de las Ciencias. (Bogotá, Colombia), v. 12, n. 1, p. 73-91, 2017. ISSN 2346-4712.

LÓPEZ, E.; GONZÁLEZ, A.; CARDOSO, M. Modelo didáctico que contribuya a la interdisciplinariedad en el proceso de enseñanza-aprendizaje de la formación inicial de los profesores de Ciencias Naturales. Órbita Científica, v. 22, n. 88, p. 134-146, 2016. ISSN 1027-4472.

MARTíneZ, A. Subjetividad, Complejidad y Educación. Psicología para América Latina, v. 12, n. 3, p. 123-134, 2010.

NÚNẼE, R. El aprendizaje de la Física en el preuniversitario y su concepción desarrolladora. Revista EDUSOL, v. 8, n. 24, p. 123-29, 2015. ISSN 1729-8091.

PACHÓN, L.; PARADA, R.; CHAPARRO, A. El razonamiento como eje transversal en la construcción del pensamiento lógico. Praxis \& Saber, v. 7, n. 14, p. 219-143, 2016. ISSN 2462 8603.

PRETZ, J. Types of intuition: inferential and holistic. UK: Edward Elgar Publishing Limited, 2011. 11.

REY, F. Advancing further the history of Soviet psychology: Moving forward from dominant representations in Western and Soviet psychology. History of Psychology, v. 17, n. 1, p. 60-78, 2014. ISSN 1939-0610

1093-4510.

REY, F. Human Motivation in Question: Discussing Emotions, Motives, and Subjectivity from a Cultural-Historical Standpoint. Journal for the Theory of Social Behaviour, v. 45, n. 4, p. 419-439, 2015.

REY, F. Subjetividad, cultura e investigación cualitativa en psicología: la ciencia como producción culturalmente situada. Liminales. Escritos sobre psicología y sociedad, n. 4, p. 13-36, 2016.

RODRIGUES, C. El rendimiento académico y su relación con el estilo de la personalidad de los estudiantes universitarios. 2011. (Máster en Psicología). Centro de Ciencias Humanas, Letras y Artes, Universidad federal de Río Grande del Norte, Biblioteca Sectorial del Centro de Ciencias Humanas, Letras y Artes.

SARRÍA, Á.; FERNÁNDEZ, D. Las invariantes de contenido en el proceso de enseñanza -aprendizaje de los Sistemas de Gestión de Bases de Datos de la Educación Preuniversitaria Revista Conrado, v. 12, n. 56, p. 243-257, 2017. ISSN 1990-8644.

SINCLAIR, M. Intuitive profile of im makers. Academy of Management annual meeting. MANAGEMENT, A. O. San Antonio, Texas: Academy of Management 2011.

SOSPEDRA, M.; ROSA, D. La formación docente universitaria en Cuba: Sus fundamentos desde una perspectiva desarrolladora del aprendizaje y la enseñanza. Estudios pedagógicos (Valdivia), v. 41, n. 1, p. 337-349, 2015. ISSN 0718-0705.

SUÁREZ, C.; DÍAZ, M.; SURI, G. Hacia un aprendizaje desarrollador en las ciencias biológicas. Cuadernos de Educación y Desarrollo, n. 31, 2013. ISSN 1989-4155.

TRIMIÑO QUIALA, C. Metodología psicodidáctica para concebir una clase desarrolladora de la personalidad. FORMACIÓN EN INVESTIGACIÓN, v. 2, n. 04, 2013. 


\section{Walfredo González Hernández}

VYGOTSKY, L. Pensamiento y lenguaje. Teoría del desarrollo cultural de las funciones psíquicas. Argentina: Ediciones Fausto., 1995.

ZEIDMANE, A.; DUKA, V. Students' Perceptions on Practical Problem Solving in Mathematics in E-environment. iJEP, v. 4, n. 2, 2014.

ZILBERSTEIN, J.; OLMEDO, S. Las estrategias de aprendizaje desde una didáctica desarrolladora. Atenas, v. 3, n. 27, p. 345-367, 2014. ISSN 1682-2749.

* Profesor titular de Ingeniería Informática da Facultad de Ciencias Ténicas, Departamento de informática, Ciudad de Matanzas, Matanzas, Cuba.

\section{Correspondência}

Walfredo González Hernández - Universidad de Matanzas. Km 3, Carretera a Varadero. CEP: 44740. Matanzas, Cuba.

E-mail:walfredogh@gmail.com

Recebido em 28 de setembro de 2017

Aprovado em 20 de março de 2018 